\title{
Elongated grains in a hopper
}

\author{
Tamás Börzsönyi ${ }^{1, \star}$, Ellák Somfai ${ }^{1, \star \star}$, Balázs Szabó ${ }^{1, \star \star \star}$, Sandra Wegner ${ }^{2}$, Ahmed Ashour ${ }^{2,3, \star \star \star \star}$, and Ralf \\ Stannarius ${ }^{2, \dagger}$ \\ ${ }^{1}$ Institute for Solid State Physics and Optics, Wigner Research Centre for Physics, Hungarian Academy of Sciences, P.O. Box 49, \\ H-1525 Budapest, Hungary \\ ${ }^{2}$ Institute of Experimental Physics, Otto-von-Guericke-University, Universitätsplatz 2, D-39106 Magdeburg, Germany \\ ${ }^{3}$ Future University, End of 90th St., Fifth Settlement, New Cairo, Egypt
}

\begin{abstract}
Flow and clogging of granular materials in a 3-dimensional hopper is investigated experimentally. We use X-ray tomography and optical methods to study this phenomenon for spherical and elongated particles. The X-ray tomograms provide information on the bulk of the hopper filling, and allow to determine the particle positions and orientations inside the silo, as well as spatial variations of the local packing density. We find that particles show a preferred orientation and thereby an enhanced order in the flowing zone of the silo. Similarly to simple shear flows, the average orientation of the particles is not parallel to the streamlines but encloses a certain angle with them. The clogged state is characterized by a dome, i. e. the geometry of the layer of grains blocking the outflow. The number of grains forming this blocking layer is larger for elongated grains compared to the case of spheres of the same volume.
\end{abstract}

\section{Introduction}

Flow and clogging of granular materials in hoppers still pose various questions which are intensively investigated nowadays [1-4]. This involves the statistics of clogging events $[2,5]$, the flow field and velocity fluctuations $[3,6,7]$, possible precursors of clogging [7], and the microscopic features of the clogged state including the visualization and analysis of the blocking forces $[1,8,9]$. Simplified model systems using spherical (or circular) particles in a confined 2-dimensional (2D) geometry have been well investigated. On the basis of these studies there is growing interest for the characterization of more general 3D systems with more common (irregular, angular, elongated) grain shapes.

There are several unsolved problems regarding flow and clogging in 3D hoppers. One of these is the influence of grain shape. Even for simple elongated grains, such as spherocylinders or ellipsoids it is not clear whether particle elongation leads to increased or decreased flow rates. Such studies typically compare particles (spherical and elongated) with equal area (2D) or volume (3D). In 2D systems Cleary and Sawley found reduced flow rates for elongated particles with nonzero friction [10, 11], while Langston found increased flow rates for elongated particles with zero friction [12] compared to the case of circles. In 3D simulations Langston found the same flow rate for

\footnotetext{
^e-mail: borzsonyi.tamas@wigner.mta.hu

$\star \star$ e-mail: somfai.ellak@wigner.mta.hu

$\star \star \star$ e-mail: szabo.balazs@wigner.mta.hu

$\star \star \star \star$ e-mail: ashour_55@yahoo.com

†e-mail: ralf.stannarius@uvgu.de
}

spheres and spherocylinders with zero friction [12], while Liu found reduced flow rates for both prolate and oblate ellipsoids with nonzero friction [13]. On the other hand, Li reported increased flow rates for oblate ellipsoids with nonzero friction [14]. The flow rate and clogging probability is strongly influenced by the orientation of the particles. In earlier studies, we systematically investigated particle rotation and average alignment in shear flows [15-20].

In the current study, we focus on the distribution of particle orientations in a 3D hopper for rod-like grains. We show that similarly to simple shear flows, the average orientation of the rods is not parallel with the streamlines, but encloses a certain angle with them. In the clogged state we charaterize the first layer of the grains above the orifice and show, that the number of grains in this shell is larger for elongated grains than for spheres of comparable volume.

\section{Experimental methods and materials}

An axisymmetric, nearly cylindrical container with a flat bottom (see figure 1a) was filled with a granular material. The diameter of the hopper was $19 \mathrm{~cm}$, its height was 21.4 $\mathrm{cm}$. At the bottom of the container, a removable plate was installed with an orifice with radius $R$. The orifice size was adjustabe by using plates with different $R$.

The granular materials investigated are shown in figures $1 \mathrm{~b}-\mathrm{d}$. The elongation of the grains is characterized by the aspect ratio $Q=L / d$. We define the equivalent radius $r_{\text {eq }}$ which is the radius of a sphere with the same volume as the particle. Three types of grains were used in the experiments presented here: 
- wooden pegs with cylinder shape and tapered ends, diameter $d=5 \mathrm{~mm}$, length $\ell=25 \mathrm{~mm}$, aspect ratio $Q=5$, equivalent radius $r_{\mathrm{eq}}=4.9 \mathrm{~mm}$

- peas, with small deviations from a perfect sphere, polydisperse with mean diameter of $7.6 \mathrm{~mm}$, standard deviation $0.23 \mathrm{~mm}, r_{\text {eq }}=3.8 \mathrm{~mm}$

- monodisperse spherical plastic (airsoft) balls with diameter of $6 \mathrm{~mm}$.
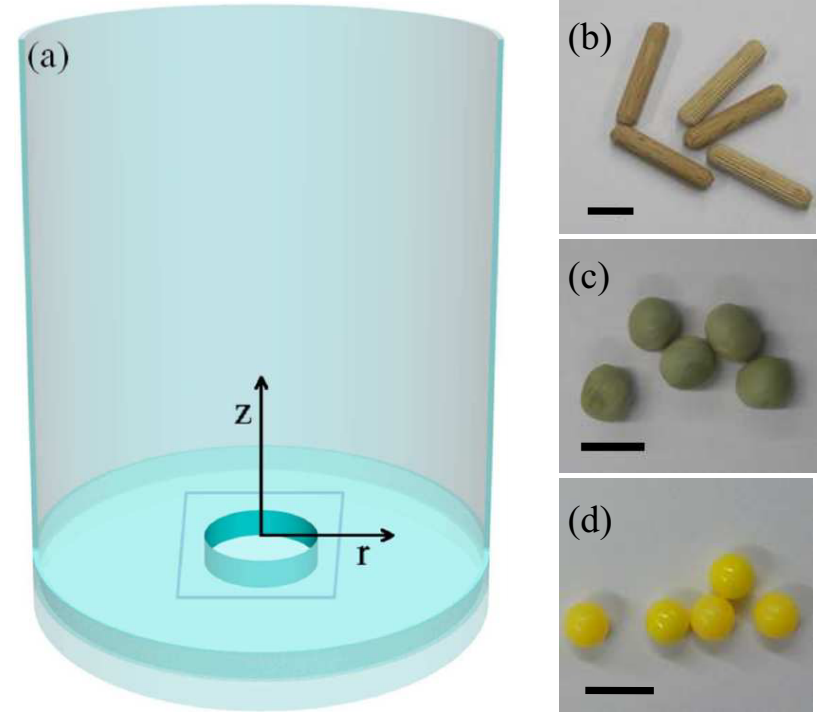

Figure 1. (a) Axisymmtertic hopper with flat bottom and circular orifice with radius $R$. (b-d) Granular materials (b) pegs with aspect ratio $L / d=5$, (c) peas and (d) plastic (airsoft) balls. The scale bars correspond to $1 \mathrm{~cm}$.

The orifice size was in the range of $1<R / r_{e q}<5$, and the clogged configuration was characterized by two methods:

(i) An X-ray tomogram (CT) of the lower half of the silo was recorded using the robot-based flat panel X-ray C-arm system Siemens Artis zeego of the STIMULATElab, Otto von Guericke University, Magdeburg. The chosen spatial resolution was $2.03 \mathrm{pixel} / \mathrm{mm}$, with recorded volumes of $25.2 \mathrm{~cm} \times 25.2 \mathrm{~cm} \times 19 \mathrm{~cm}$. The $3 \mathrm{D}$ arrangement of the particles was then determined from the X-ray tomogram.

(ii) A digital camera was placed close to the orifice and the number of grains forming the last (clogging) layer was determined by image processing.

\section{Results and discussion}

For the experiments, the silo was filled with a closed orifice, then the orifice was opened and the granular flow was running until the system clogged. This clogged configuration was investigated (CT or digital camera), and thereafter the system was perturbed at the orifice to generate flow again. About 30 configurations were recorded for each material. First, we will show data obtained with CT for pegs, focusing on grain orientation, density distribution and ordering, then we will analyze data obtained by a digital camera for all three materials focusing on the number of grains which build up the last layer (dome) which blocked the flow.

Vertical cross sections (central slice) of two subsequent tomograms are presented for pegs in figure 2 . The first tomogram was taken after several avalanches, thus the orientation and packing of the grains reflects the properties

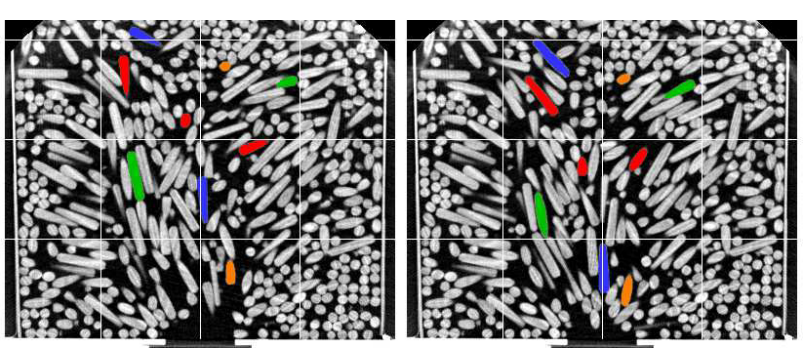

Figure 2. Vertical cross sections of two subsequent tomograms (two clogged states separated by a single avalanche) for pegs. In the flowing region particles are aligned. The pictures show the lower $16 \mathrm{~cm}$ of the nearly cylindrical hopper, a rectangular mesh of $5 \mathrm{~cm} \times 5 \mathrm{~cm}$ has been superimposed to guide the eye. A few colored grains indicate the displacement of selected particles between the two subsequent clogged states. The diameter of the hopper outlet was $35 \mathrm{~mm}$.

of the developed flow and alignment (initial conditions erased in the flowing regions). As explained above, these tomograms were taken in the clogged state. During the discharge between these two tomograms, approximately 60 particles left the hopper. The displacement of the material due to the flow is visualized by a few selected particles which are marked with colors in figure 2 . It is clearly visible that the central region moves faster, especially near the outlet. Stagnant zones can be identified near the walls especially at the bottom of the hopper. In the flowing region particles are expected to be oriented nearly (but not exactly) parallel to the streamlines [15-17]. In order to quantify the ordering and alignment of the particles and to determine the flow field and the density distribution, tomograms from 26 subsequent clogged states have been analyzed. In figures $3(\mathrm{a})-(\mathrm{b})$, the color maps visualize the density distribution, and the average alignment angle $\left(\theta_{\mathrm{av}}\right)$ of the particles in the $r-z$ plane. The alignment angle is measured with respect to the streamlines, which are indicated by white lines. The streamlines were calculated using the direction of average particle displacement between subsequent clogs. In order to better visualize the correlation between flow and orientation, on figure $3 \mathrm{a}$ we represented the average orientation of the particles by ellipses. The order parameter is represented by the flattening of the ellipses, $\mathrm{S}=1$ (perfect order) would be represented by a thin line, and $\mathrm{S}=0$ (isotropic state) by a circle [4].

Altogether, figures $3 a-b$ show that in the flowing (sheared) regions we find well oriented rods due to the shear flow, while near the walls tangential alignment is preferred due to the boundary-induced ordering. We see, that in the shear flow the average orientation is not parallel to the streamlines, but there is a flow alignment angle as 

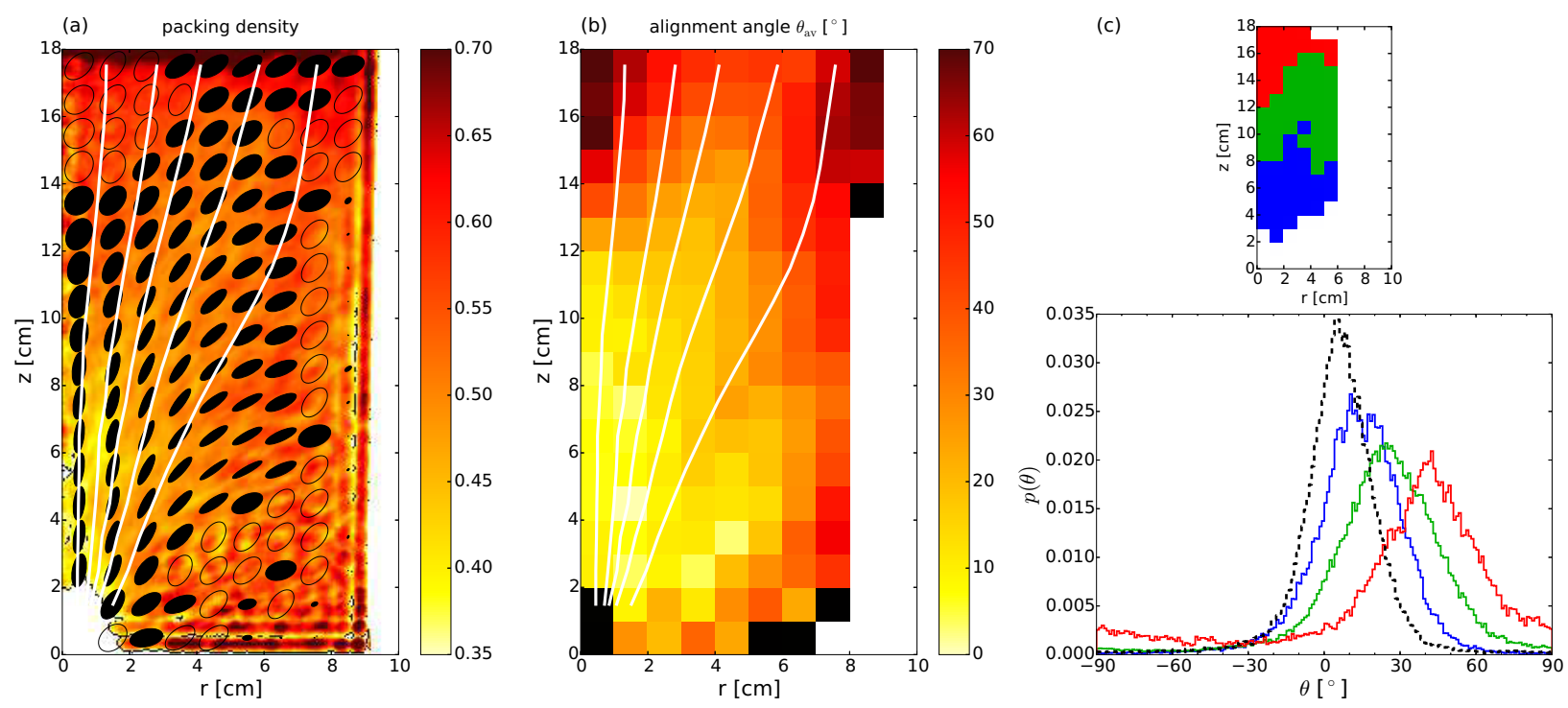

Figure 3. Distributions of (a) packing density, (b) and average alignment angle $\theta_{\mathrm{av}}$ in the $r-z$ plane. Streamlines are determined from particle displacements between subsequent tomograms and are indicated by white lines. The alignment angle is measured with respect to the flow direction. On panel (a) the alignment and ordering of the particles is represented by ellipses (see text). Data have been calculated from 26 subsequent tomograms (examples shown in figure 1). (c) Distribution of particle orientations $\theta$ with respect to the streamlines in three regions as indicated in the top panel. The dashed line corresponds to a stationary distribution in a shear flow [16].

expected [15-17]. The sheared regions are characterized by lower packing fraction, consistent with our earlier observation in shear flow, where the shear induced dilation was found to be only partially compensated by a slight density increase due to the ordering of the grains [19].

In order to characterize the evolution of the grain alignment angle during flow, we plotted the distributions of $\theta_{\mathrm{av}}$ in three different regions of the hopper in figure $3 \mathrm{c}$. The three regions are marked with different colors (see top panel of figure 3c). The corresponding 3 curves show gradual narrowing of the angular distribution and decreasing of the average angle as the material is exposed to larger and larger deformation as it is displaced downwards in the hopper. In figure $3 \mathrm{c}$ we also show the distribution of angles of the same particles in stationary split bottom Couette flow (dashed line) [20]. We see that near the outlet (blue curve) the angular distribution almost reached the case of stationary shear.

In the following we characterize the arrangement of particles in the first layer of grains (above the orifice). In 2D a continuous force chain from one side of the orifice to the other can be used to identify the particles that form the blocking arch. The definition of the first layer is not straightforward in $3 \mathrm{D}$, one can only roughly determine the blocking layer of particles. Of course, not only the grains in that first layer contribute to the clog, i. e. removal of other particles can also destabilize the blocked state, in 3D as well as in $2 \mathrm{D}$. In our 3D experiments, we roughly define the blocking particles by selecting the minimal number of particles that form a closed shell above the outlet which cannot be penetrated by particles above that layer. A reasonable assumption for the mean number $N$ of monodis- perse spheres of radius $r=r_{e q}$ blocking an orifice with radius $R$ can be estimated when one compares the area of a hemisphere (dome) of radius $R+r_{e q}$ with the cross section area of each grain, $\pi r_{e q}{ }^{2}$. This yields $N \approx 2 \gamma\left(R / r_{e q}+1\right)^{2}$ The parameter $\gamma$ is an area-filling factor which accounts for the fact that the grains can be displaced out of the hypothetical hemisphere. One may expect that $\gamma$ is of the order of one, larger than the maximum packing fraction of circles in a plane, 0.906. The number of particles identified in the first layer of the clog are presented as a function of $R$ in figure 4 for spherical particles and rods, respectively. Data for peas and monodisperse smooth plastic spheres collapse to the same curve with $\gamma \approx 1.03$. Thus for these samples differences in particle properties like detailed geometrical shape and friction play only an insignificant role. For the case of cylindrical particles (figure 4) the growth of $N$ with increasing $R$ is faster with $\gamma \approx 1.21$. This is a consequence of grain alignment, i.e. that grains forming the blocking shell are preferentially oriented such that longer axes are nearly perpendicular to the local shell surface. Thus, the shell is thicker (in units of the equivalent radius) for elongated grains, one needs more cylinders to form the blocking shell than spheres of the same equivalent radius.

We are currently working on the characterization of clogging statistics and hopper flow rate as a function of particle elongation for rod-like particles [21] in a 3D hopper. Characterization of the flow field as a function of grain shape in a quasi-2D hopper is also in progress. For elongated grains the profile of the vertical velocity clearly deviates from gaussian, and tunnel flow occurs [22]. 


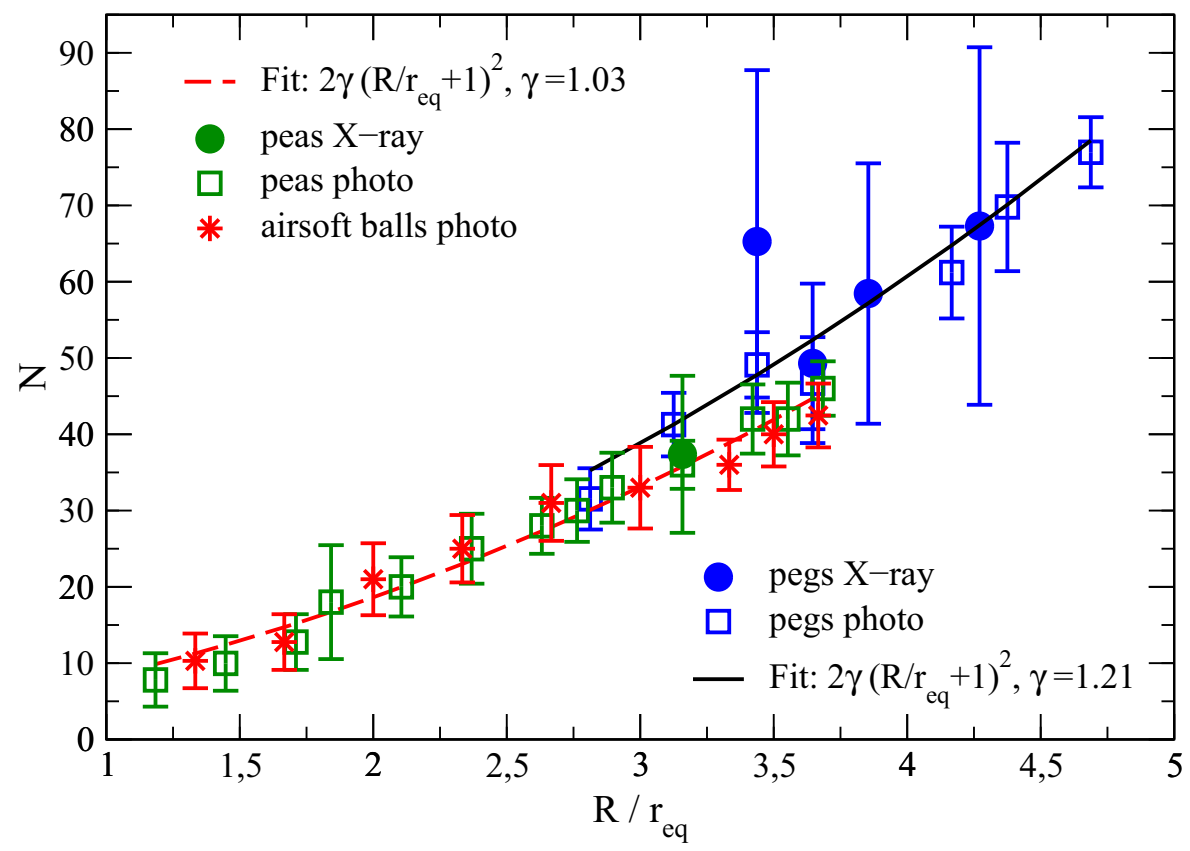

Figure 4. Number of particles forming the blocking dome structure as a function of the orifice radius for peas, airsoft balls and pegs. The fits with Eq. (1) result $\gamma \approx 1.03$ and $\gamma \approx 1.21$ for beads and rods, respectively. Each datum point is an average of 30 measurements.

\section{Acknowledgements}

We thank G. Rose and the STIMULATE-lab of Otto von Guericke University Magdeburg for the opportunity to use the X-ray CT facilities (funded by BMBF, reference No. 03IP710) and support. Financial support from the DAAD/MÖB researcher exchange program (Grants No. 57141022 and 64975), the Hungarian Scientific Research Fund (Grant No. OTKA NN 107737) and the János Bolyai Research Scholarship of the Hungarian Academy of Sciences are acknowledged.

\section{References}

[1] J. Tang and R.P. Behringer, EPL 114, 34002 (2016)

[2] C. C. Thomas and D. J. Durian, Phys. Rev. Lett. 114, $178001(2015)$

[3] S.M. Rubio-Largo, A. Janda, D. Maza, I. Zuriguel, and R.C. Hidalgo Phys. Rev. Lett. 114, 238002 (2015)

[4] T. Börzsönyi, E. Somfai, B. Szabó, S. Wegner, P. Mier, G. Rose, and R. Stannarius, New J. Phys. 18, 093017 (2016)

[5] I. Zuriguel, A. Garcimartín, D. Maza, L. A. Pugnaloni, and J. M. Pastor, Phys. Rev. E 71, 051303 (2005)

[6] S. Tewari, M. Dichter and B. Chakraborty, Soft Matter 9, 5016 (2013)

[7] C. C. Thomas and D. J. Durian, Phys. Rev. E 94, 022901 (2016)

[8] J. Tang and R.P. Behringer, CHAOS 21, 041107 (2011)

[9] R.C. Hidalgo, C. Lozano, I. Zuriguel, and A. Garcimartín, Granular Matter 15, 841-848 (2013)
[10] P.W. Cleary. Second International Conference on CFD in the Minerals and Process Industries, pages 7176, 1999.

[11] P.W. Cleary and M.L. Sawley. Applied Mathematical Modelling 26, 89 (2002)

[12] P.A. Langston, M.A. Al-Awamleh, F.Y. Fraige, and B.N. Asmar. Chem. Eng. Sci. 59, 425 (2004)

[13] S.D. Liu, Z.Y. Zhou, R.P. Zou, D. Pinson, and A.B. Yu. Powder Technology 253, 70 (2014)

[14] J. Li, P.A. Langston, C. Webb, and T. Dyakowski. Chemical Engineering Science 59, 59172004.

[15] T. Börzsönyi, B. Szabó, G. Törös, S. Wegner, J. Török, E. Somfai, T. Bien, and R. Stannarius, Phys. Rev. Lett. 108, 228302 (2012)

[16] T. Börzsönyi, B. Szabó, S. Wegner, K. Harth, J. Török, E. Somfai, T. Bien, and R. Stannarius, Phys. Rev. E 86, 051304 (2012)

[17] S. Wegner, T. Börzsönyi, T. Bien, G. Rose, and R. Stannarius, Soft Matter 8, 10950-10958 (2012)

[18] T. Börzsönyi and R. Stannarius, Soft Matter 9, 7401 (2013)

[19] S. Wegner, R. Stannarius, A. Böse, G. Rose, B. Szabó, E. Somfai, and T. Börzsönyi, Soft Matter 10, 5157 (2014)

[20] B. Szabó, J. Török, E. Somfai, S. Wegner, R. Stannarius, A. Böse, G. Rose, F. Angenstein, and T. Börzsönyi, Phys. Rev. E 90, 032205 (2014)

[21] A. Ashour, S. Wegner, T. Trittel, T. Börzsönyi, and R. Stannarius, Soft Matter 13, 402 (2017)

[22] B. Szabó, E. Somfai, S. Wegner, A. Ashour, D. Fischer, R. Stannarius, and T. Börzsönyi, In preparation. 